Katharina Schmidt* ${ }^{*}$ and David Hochmann

\title{
Characterization of loading profiles via handrails and harness during body weight supported treadmill training
}

\begin{abstract}
Body weight supported treadmill training is used for patients who are severely limited in their movements. During walking therapy handrails and harnesses ensure the patient's stability and safety. Although, locomotion training is a common rehabilitation technique, data of forces applied to handrails or overhead lifts are limited. Therefore, the primary objective of this study was to measure the vertical reaction force during treadmill therapy. An additional purpose was the characterization of the loading profiles by defining suitable parameters.

To measure the patient's needs for support during treadmill training, the handrails were instrumented with strain gauges and a force sensor was placed between the harness and the ceiling lift. In order to provide gait phase detection, an inertial sensor was attached to each foot.

The results confirm that the combined measuring systems are appropriate to record the total vertical weight relief. Although all patients show different loading profiles, a recurring pattern can be recognized for each patient, which allows the derivation of various parameters to characterize the single profiles. The additional information of the measurements can help the supervising physiotherapist to better understand the specific level of support the patient needs. A subsequent response and the providing of corresponding instructions by the therapist can lead to an even more individualized therapy.
\end{abstract}

Keywords: weight relief, body weight support, treadmill training, force measurement, exoskeleton.

https://doi.org/10.1515/cdbme-2021-2088

\footnotetext{
*Corresponding author: Katharina Schmidt: Münster University of Applied Sciences, Biomechatronics Research Laboratory, Bürgerkamp 3, 48565 Steinfurt, Germany, e-mail: schmidt.katharina@fh-muenster.de
}

2nd Author David Hochmann: Münster University of Applied Sciences, Biomechatronics Research Laboratory, Bürgerkamp 3, 48565 Steinfurt, Germany

\section{Introduction}

Spinal cord injuries (SCIs) can have a variety of traumatic and non-traumatic causes. Both occurrences lead to temporary or persistent damage to the spinal cord and consequently to a restriction of the patient's movement capabilities. For restoring the walking ability, different therapeutic approaches of treadmill training with or without body weight support (BWS) are available. Especially the body weight supported treadmill training (BWSTT) is a well-known rehabilitation technique for patients who are not able to walk independently. In this case, handrails and an overhead lift can provide weight relief and increase the patient's safety and stability.

The comparison of treadmill training with or without BWS has been subject of previous studies [1]. Besides, the effect of various percentages of BWS provided by an overhead harness during walking has been examined for patients with stroke [2] and Parkinson's disease [3]. However, all these investigations focused on the impacts of different therapeutic approaches on functional outcome measurements like walking speed, endurance, or kinematic data. To our knowledge, the BWS itself has not been analyzed.

A similar situation exists for handrail studies, where even less data is available for handrail loads during gait. Although Komisar et al. acquired handrail force data during walking, the analysis was related to the effects of handrail height and participant age on trunk and shoulder kinematics when recovering from perturbations [4]. Similarly, all other studies mainly comprised the influence of different handrail positions and designs, whereby the analysis of the forces applied to the handrails is limited to the sit-to-stand movement [5] or different falling motions after unexpected balance loss [6]

Consequently, data acquisition is still limited and a detailed evaluation of the patient's weight relief during treadmill training is missing. To fill this existing data deficit, one purpose of the present study is the combined measurement of the vertical forces applied by the patient to both BWS systems, the handrails, and the overhead lift. A subsequent force data analysis of the BWSTT aims to identify parameters to characterize the loading profiles, which can result in a better understanding of the patient's need for support. 


\section{Methods and Materials}

The BWSTT with the Hybrid Assistive Limb (HAL), especially the Lower Limb Model, was chosen as an exemplary object of examination. The training setup includes the treadmill, side handrails, and a vertically adjustable overhead lift (see Figure 1 a).

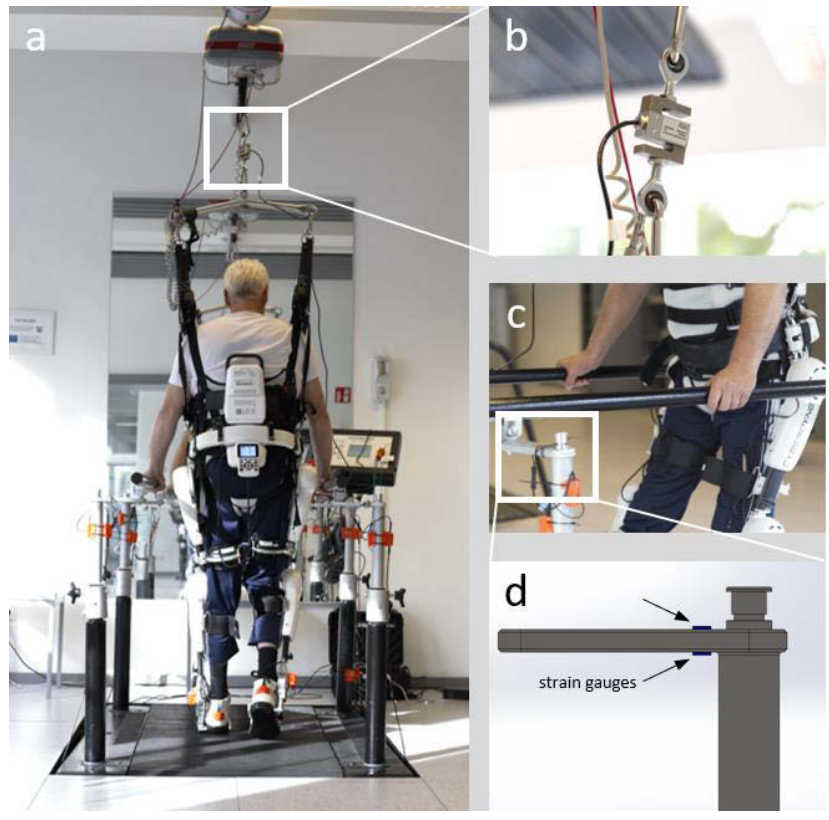

Figure 1: Measurement setup of the HAL training (a) including the force sensor of the overhead lift (b) and the instrumented handrails (c) with the applied strain gauges (d).

To measure the patient's needs for overhead support, the 1 -axis force sensor KD9363s (ME-Meßsysteme $\mathrm{GmbH}$ ) was placed between the spreader bars of the harness and the ceiling lift (see Figure $1 \mathrm{~b}$ ). All four handrail bases were instrumented with strain gauges, whereby the connection as a full bridge enables the distinction between pushing down or pulling up the handrail (see Figure $1 \mathrm{c}-\mathrm{d}$ ). The individual measuring points were calibrated with weights up to 30 kilograms in tension direction for the lift sensor and in pushing direction for the handrails. The forces applied on each handrail side result from the sum of the individually measured forces on each base.

In addition to the load sensors, an inertial sensor was attached to the heel of each shoe. The data analysis of the integrated accelerometer and gyroscope enabled gait phase detection.

Before each measurement, the sensors for load measurements were tared and additionally, for parallel recording via our self-developed system, all sensors were synchronized by cable. The acceleration and angle-velocity of the inertial sensor were recorded at a measuring frequency of $70 \mathrm{~Hz}$, whereby all sensors for loading measurements captured the data at a sample rate of $1000 \mathrm{~Hz}$. For data processing and analysis Python 3.7 was used. Due to data loss, the recorded force data was resampled to a frequency of $200 \mathrm{~Hz}$ and filtered by a zero-phase low pass Butterworth $2^{\text {nd }}$ order filter with a cut-off frequency of $10 \mathrm{~Hz}$.

In total, the loading profiles of $14 \mathrm{HAL}$ patients (13 male) were recorded, including 4 follow-up patients who completed their regular therapy earlier. The SCI of all patients was caused by trauma and is located at a level between C2 and L3.

\section{Results}

The combined measurement of the different sensors allows to capture the vertical weight relief. The measurements of Patient A (see Figure 2) and Patient B (see Figure 3) were taken as examples for the different loading profiles, which represent typical courses from the available data of the 14 recorded patients. Both selected patients were male and recently started training, whereby Patient A (29 y, $73.4 \mathrm{~kg})$ has an incomplete T8-SCI and Patient B (40 y; $74.3 \mathrm{~kg})$ an incomplete T12-SCI.
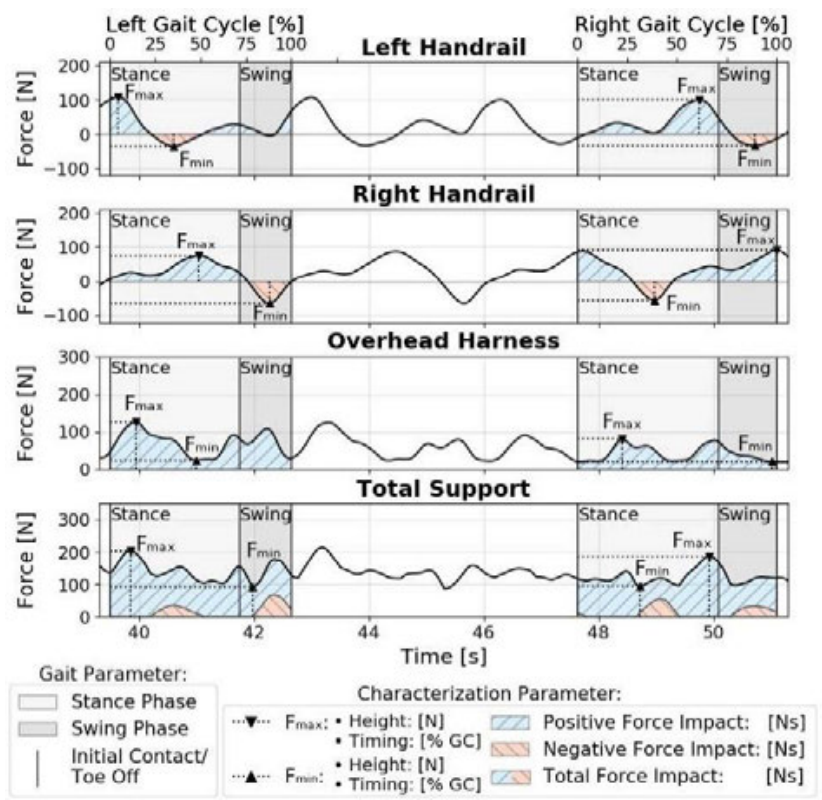

Figure 2: Vertical forces applied to the support systems during treadmill training from Patient $A$ and graphical illustration of the temporal and force characterization parameters exemplary for the left and right gait cycle (GC).

Figure 2 illustrates the applied vertical forces to the BWS systems. For the handrails, positive values represent pushing forces and negative values pulling the handrail upwards. Only vertical tension forces can be measured for the overhead lift. In addition to these three individual force curves, the total 
support is depicted, which results from the sum of the absolute force values of all single BWS systems. The magnitude ensures that negative values due to pulling the handrail do not reduce or eliminate the total support needed. Based on the gait phase detection, the individual gait cycles and phases can be highlighted in the temporal progression by the second upper $\mathrm{x}$ axis.

By analyzing the curves, parameters for characterization can be derived. These include both, the time and force curves. The minimum and maximum force values for each gait cycle give information about the amount of support needed, whereby corresponding peak timings identify the point in time when the patient needs the respective support. In addition to the peak heights, the duration of the applied forces is also relevant. This parameter is included in the calculation of the force impact, which represents the area under the force curve. In this way, a characterization can be determined for both, the left and right gait cycle.

Table 1: Characterization parameters (Mean, SD) of Patient A for the left gait cycle.

\begin{tabular}{|c|c|c|c|c|c|c|c|}
\hline & \multicolumn{2}{|c|}{$\begin{array}{c}\text { Peak-Height } \\
{[\mathrm{N}]}\end{array}$} & \multicolumn{2}{|c|}{$\begin{array}{l}\text { Peak-Timing } \\
{[\% \text { GC }]}\end{array}$} & \multicolumn{3}{|c|}{$\begin{array}{l}\text { Force Impact } \\
{[\text { [Ns] }}\end{array}$} \\
\hline & Max. & Min. & Max. & Min. & Pos. & Neg. & Total \\
\hline Left & 107.5 & -23.8 & 14.6 & 43.5 & 92.7 & -13.4 & 106.1 \\
\hline Handrail & 7.1 & 12.0 & 22.1 & 10.3 & 16.4 & 7.9 & 13.9 \\
\hline Right & 80.3 & -69.8 & 51.9 & 86.1 & 89.8 & -33.3 & 123.1 \\
\hline Handrail & 7.9 & 12.9 & 1.7 & 15.6 & 16.1 & 9.2 & 12.2 \\
\hline Overhead & 69.6 & 15.3 & 43.5 & 60.0 & 116.1 & & 116.1 \\
\hline Harness & 32.3 & 5.3 & 30.2 & 34.5 & 54.0 & - & 54.0 \\
\hline Total & 164.9 & 57.9 & 42.0 & 77.7 & 299.7 & -47.7 & 347.4 \\
\hline Support & 25.5 & 17.3 & 41.4 & 11.4 & 49.9 & 15.9 & 59.4 \\
\hline
\end{tabular}

Table 1 contains the characterization parameters exemplary for the left gait cycle for the presented training unit of Patient A. During this gait section, the patient completed a total of 66 gait cycles, whereby the first and last are not included in the statistical calculation. A total of 7 parameters can be derived for each single handrail support system and the total support needed. Due to the limited force measurement of the harness support in only one direction, the negative impact can not be evaluated. Consequently, for this curve the positive and total force impact are equal.

Patient A demonstrates a minor variation in the peak height and force impact of the handrail data. This emphasizes a constant and recurring need for support over the gait section. However, noticeable are the differences in the support method, whereby the patient applies stronger traction forces to the right handrail in order to be able to swing the left leg forward. In contrast, nearly no support via the harness is needed. Therefore, a major standard deviation is present for this parameter since the maxima and minima are distributed over the entire gait cycle. This increased variation is also represented in the total support, whereby the consideration of the force impact illustrates that each individual BWS system has an approximately equal influence.
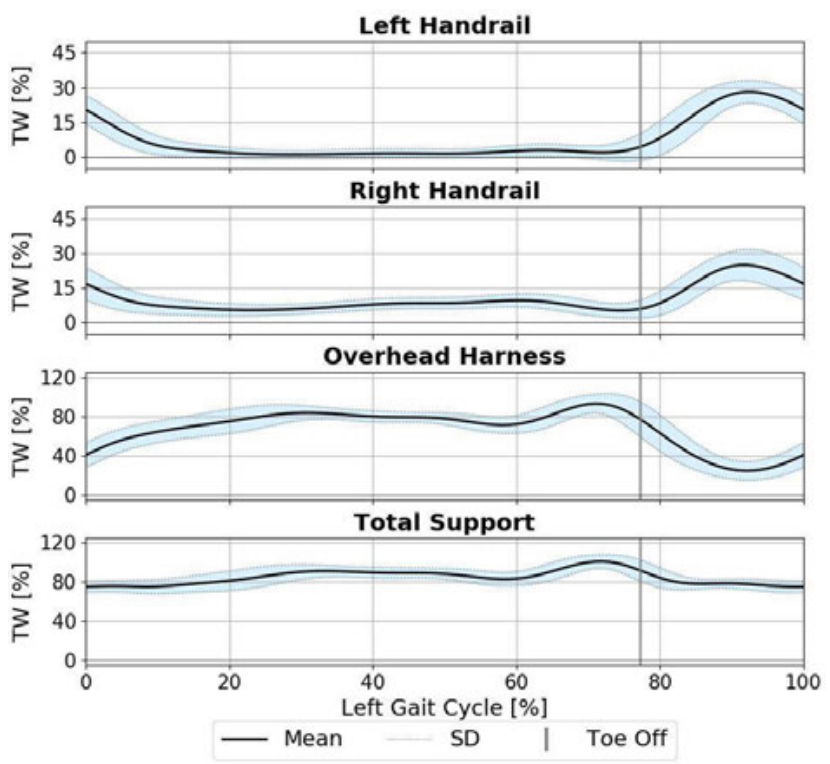

Figure 3: Mean and standard deviation of the loading profile over the left gait cycle of Patient $B$ as a percentage of total weight (TW) including body weight and exoskeletal equipment ( $n=483$ gait cycles; Mean cycle duration: $3.4 \mathrm{~s}$; Mean percentage of stance phase: $77.3 \%$; Treadmill velocity: $1.2 \mathrm{~km} / \mathrm{h}$ ).

The loading profile of Patient B is shown in Figure 3. In this case the load was normalized to the percentage of the total weight, which is composed of the patient body weight and the weight of the HAL including the harness and safety devices (approx. $17.5 \mathrm{~kg}$ ). It is displayed as a mean value curve including standard deviation over the left gait cycle. The minor standard deviation clarifies that a recurring pattern is generated. Thus, a repetitive loading pattern can be recognized for all other recorded patients.

A comparison of the depicted loading profiles of the two patients demonstrates clear differences in the curve progression. Patient B requires a relatively high level of support. The minor support phases via the harness during the swing phase are compensated by pushing on the handrails. This results in a nearly constant level of total support over the entire gait cycle between $80 \%$ to $100 \%$ weight relief. In contrary, the support demand of Patient A is less and contains a change of sign in the force data of the handrails. 


\section{Discussion and Outlook}

This research study is the first to provide a parallel measurement of the weight relief via handrails and an overhead lift during BWSTT. The presented results and loading profiles confirm the suitability of the measurement systems. The additional information provided by the described parameters can identify the level and timing of the support needed by the patient during the gait cycle. Especially, the variations between the loading profiles of the two handrail sides from Patient A demonstrates that the partially paretic muscle functions are compensated differently. Consequently, this is the point where therapeutic intervention can be initiated to selectively strengthen these muscle groups and individualize the training according to the needs of the patient.

Due to the given training setting, only the measurement of the vertical force components could be implemented without changing elements of the lift or handrails. The forces applied to the handrails in the medio-lateral and anterior-posterior directions can not be measured. Therefore, it may be possible that a patient's total support needs are greater when additional forces are applied to the handrails in the other directions.

Besides, it is noted that the load sensor calibrations were performed in only one direction and with weights up to 30 kilograms. Accordingly, the measured loads are partly outside the calibration range and the accuracy of these values is not validated.

Since the ratio of SCIs due to trauma is high compared to other causes, additional patient restrictions are often present. A high spinal fracture in the cervical spine area can lead to movement restrictions of the upper extremities, which require fixation of the hands to the rails. Hence, the handrails usually bear only the self-weight of the patient's hand, which results in a limited analysis of handrail forces. For patients who require manual assistance by a physiotherapist to swing one limb forward, it was necessary to ensure that only a minor degree of assistance is provided, which does not change the level of BWS. Otherwise, the subjects were excluded. For other supplementary diagnoses, such as ankle fractures, the patient may not bear their entire body weight on their feet while walking. In this case, the measured overhead and handrail support can provide feedback on how much weight is offloaded from each foot during the stance phase.

Another advantage of the measurements can be an automated documentation of a patient's training progress. Initial recordings of the patient's loading profile at different times demonstrate that changes between single training sessions are detectable. This would give the additional opportunity to compare different BWSTTs regarding changes in the loading profiles. Also, other parameters can be included in this evaluation, such as the number of steps or the symmetry of the gait pattern, which can be provided by a detailed analysis of the inertial sensor data. But at this point further measurements and statistical analysis are necessary.

However, the additional information provided by the measurement systems can characterize the individual needs of the patient and can lead to an even more precisely defined therapy. Even though the measurements are exemplary based on HAL training, they can also be extended to other types of therapy.

\section{Acknowledgement}

The authors acknowledge the financial support by the Care Center Deutschland GmbH. Special thanks to the Cyberdyne CareRobotics $\mathrm{GmbH}$, where this study took place.

\section{Author Statement}

Research funding: This study received funding from the Care Center Deutschland GmbH. The funder was not involved in the study design, collection, analysis, or interpretation of data. Conflict of interest: Authors state no conflict of interest. Informed consent: Informed consent has been obtained from all individuals included in this study. Ethical approval: The research related to human use complies with all the relevant national regulations and institutional policies.

\section{References}

[1] Visintin M, Barbeau H, Korner-Bitensky N, Mayo NE. A new approach to retrain gait in stroke patients through body weight support and treadmill stimulation. Stroke; 1998: 29(6):1122-1128.

[2] Sousa CO, Barela JA, Prado-Medeiros CL, Salvini TF, Barela AM. The use of body weight support on ground level: an alternative strategy for gait training of individuals with stroke. Journal of NeuroEngineering and Rehabilitation; 2009:6(1):1-10.

[3] Atan T, Taşkiran ÖÖ, Tokçaer AB, Karataş GK, Çalişkan AK, Karaoğlan B. Effects of different percentages of body weightsupported treadmill training in Parkinson's disease: a doubleblind randomized controlled trial. Turkish journal of medical sciences; 2019:49(4):999-1007.

[4] Komisar V, Novak AC. Effect of handrail height and age on trunk and shoulder kinematics following perturbation-evoked grasping reactions during gait. Human factors; 2021: 00187208211013631.

[5] Kato T, Sekiguchi Y, Honda K, Izumi SI, Kanetaka H. Comparison of handrail reaction forces between two different handrails during sit-to-stand movement in the elderly. Clinical Biomechanics; 2020:80:105130.

[6] Komisar V, Nirmalanathan K, King EC, Maki, BE, Novak AC. Use of handrails for balance and stability: characterizing loading profiles in younger adults. Applied ergonomics; 2019:76:20-31. 